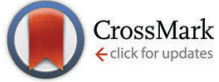

Cite this: Phys. Chem. Chem. Phys., 2016, 18, 11184

Received 14th January 2016, Accepted 22nd March 2016 DOI: $10.1039 / c 6 c p 00307 a$

www.rsc.org/pccp

\title{
Facilitated diffusion in the presence of obstacles on the DNA
}

\begin{abstract}
David Gomez ${ }^{\star a}$ and Stefan Klumpp ${ }^{\text {ab }}$
Biological functions of DNA depend on the sequence-specific binding of DNA-binding proteins to their corresponding binding sites. Binding of these proteins to their binding sites occurs through a facilitated diffusion process that combines three-dimensional diffusion in the cytoplasm with one-dimensional diffusion (sliding) along the DNA. In this work, we use a lattice model of facilitated diffusion to study how the dynamics of binding of a protein to a specific site (e.g., binding of an RNA polymerase to a promoter or of a transcription factor to its operator site) is affected by the presence of other proteins bound to the DNA, which act as 'obstacles' in the sliding process. Different types of these obstacles with different dynamics are implemented. While all types impair facilitated diffusion, the extent of the hindrance depends on the type of obstacle. As a consequence of hindrance by obstacles, more excursions into the cytoplasm are required for optimal target binding compared to the case without obstacles.
\end{abstract}

\section{Introduction}

Processing of the genetic information is to a very large extent dependent on the sequence-specific binding of proteins to DNA. Examples include binding of transcription factors to specific operator sites and binding of RNA polymerases to promoters. ${ }^{1}$ Sequence-specificity is typically not perfect, and binding motifs therefore are typically 'fuzzy', with a range of different sequences displaying comparable affinities for the protein and an even larger range showing weak affinity. ${ }^{2}$ In addition, binding of DNA-binding proteins (DBPs) to DNA usually has an electrostatic component that is independent of the sequence, i.e. non-specific. For that reason both the equilibrium binding pattern and the binding kinetics of DBPs can be quite complex. ${ }^{3}$ Nevertheless, DBPs find their functional binding sites (of size $\sim 10-30 \mathrm{bp}^{1,4}$ ) with remarkable efficiency despite the large number of non-specific binding sites $\left(\sim 10^{6}\right.$ in the genome of $E$. coli) that compete for binding with the functional site. In a seminal work, Riggs et al. measured the in vitro rate at which the lac repressor finds its promoter, and they found that under certain conditions, the lac repressor binds to its target at faster rates than the simple three dimensional diffusion limit. ${ }^{5}$ To explain this phenomena, Berg, Winter and von Hippel proposed the so called facilitated diffusion model, ${ }^{6,7}$ in which such high binding rates are achieved

\footnotetext{
${ }^{a}$ Max Planck Institute of Colloids and Interfaces, Science Park Golm, 14424 Potsdam, Germany. E-mail: david.gomez@mpikg.mpg.de; Tel: +49331 567-9622

${ }^{b}$ Institute for Nonlinear Dynamics, Georg-August University Göttingen, Friedrich-Hund-Platz 1, 37077 Göttingen, Germany
}

if the DBP undergoes a combination of three-dimensional (3D) excursions in the bulk solution, together with one-dimensional (1D) sliding on the DNA. This theoretical model has been strongly supported by experimental techniques that directly showed the number of basepairs scanned via 1D sliding, ${ }^{8-10}$ and the average fraction of time the DBP remains bound to the DNA before unbinding. ${ }^{11,12}$

Since it was proposed, facilitated diffusion has been the subject of much theoretical, ${ }^{13-18}$ experimental ${ }^{4,19-24}$ and computational $^{25-27}$ efforts. Interest in facilitated diffusion has been renewed by the direct observation of facilitated diffusion in bacterial cells using single-molecule techniques. ${ }^{11,12}$ One key feature that is different in cells compared to in vitro is that the cytoplasm is not a dilute solution, but a rather crowded environment that can be occupied up to $40 \%$ by macromolecules. ${ }^{28,29}$ The presence of these macromolecules (crowders) inside the cells has effects on diffusion, ${ }^{30-32}$ enzymatic reactions, ${ }^{33-36}$ protein folding ${ }^{37-40}$ and gene expression. ${ }^{41-43}$ In addition, the DNA itself is also covered with proteins that bind to the DNA in order to perform functions such as transcription, DNA repair and gene regulation. ${ }^{1,44-46}$ Moreover, the DNA itself is spatially organized and compacted by histones in eukaryotes and nucleoid-associated proteins in bacteria. ${ }^{47,48}$ Thus, the DNA is also highly occupied $(\sim 30 \%)$ by DBPs that affect the facilitated diffusion process. So far, only a few of the theoretical studies have included the impact of these macromolecules on the promoter finding dynamics. ${ }^{49-53}$

In this paper we address the effects of obstacles bound to the DNA in facilitated diffusion, i.e. in the promoter search of RNA polymerase or the search of a transcription factor for its functional binding site. We use a lattice model to study the 
facilitated diffusion, first in the free case without crowders and then in the presence of obstacles on the DNA. Specifically, we consider three different types of obstacles characterized by different dynamics on the DNA. We show that, for all types, facilitated diffusion is impaired when the DNA occupation fraction increases, but the strength of the effect depends on the type of obstacle. The lattice model we use aims to create a clear conceptual understanding of the facilitated diffusion rather than to provide a detailed computational description of the process. The method is, however, applicable more generally.

\section{Lattice model for simulating facilitated diffusion}

Facilitated diffusion is studied using Monte Carlo simulations of particles on a 3D lattice with periodic boundary conditions. The simulation box has a total volume $V=m_{x} \times m_{y} \times m_{z} l^{3}$, where $l$ is the lattice spacing, chosen as the linear extension of the smallest particle type. A DNA molecule is implemented as a linear arrangement of lattice sites along the $y$ axis with $x=z=$ $\left(m_{x} / 2\right) l=\left(m_{z} / 2\right) l$. The system contains a target (e.g., a promoter or a regulatory binding site) and two types of particles: One DBP searching for the target and obstacles on the DNA, see Fig. 1A. All particles are taken to occupy exactly one lattice site. We take the target to be static, and its position is randomly chosen among the DNA lattice sites. The DBP is initially placed randomly in the bulk solution, whereas the obstacles are placed on DNA lattice sites, occupying a fraction of DNA equal to $O / \mathcal{L}$, where $O$ is the number of obstacles and $\mathcal{L}=m_{y}$ the length of the DNA template. Here, obstacles are considered to be only on the DNA and they are not allowed to unbind and diffuse in the bulk solution.

In our implementation, the DBP can either diffuse in the bulk solution or slide on the DNA. If the DBP is in the bulk solution, at each simulation time step (of duration $\tau$ ), it moves to each neighboring site with a probability of $1 / 6$. Thus, DBP diffuses in the bulk solution with diffusion constant $D_{3}=l^{2} /(6 \tau)$. If one of the neighboring sites is part of the DNA and it is free, the move is always accepted, and the DBP is placed onto the DNA. In contrast, if the DNA site is occupied by an obstacle, the move is rejected and the DBP stays in the bulk solution
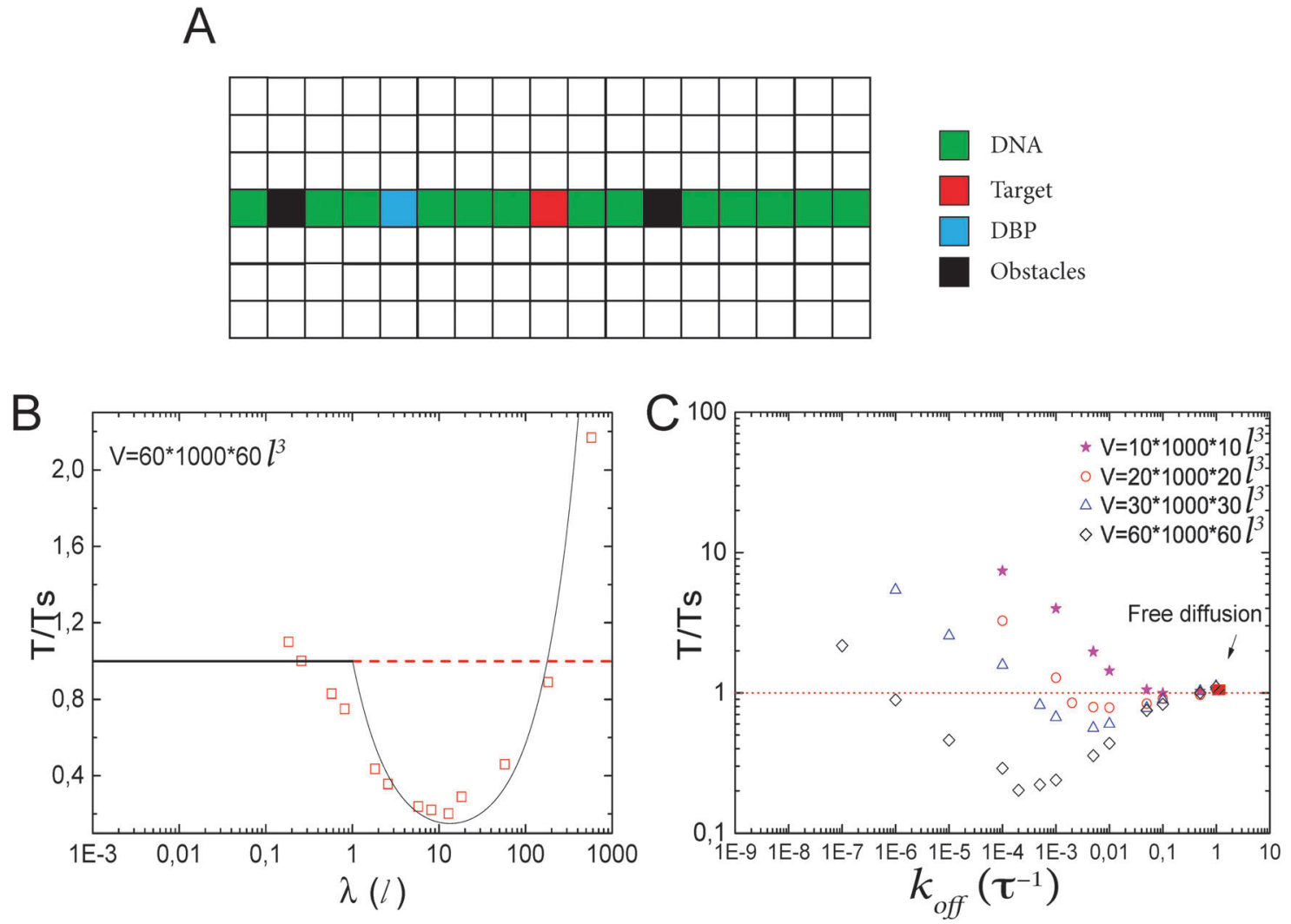

Fig. 1 Lattice model for facilitated diffusion: (A) schematic view of the model. DBP (light blue) diffuses by hopping either to one of the six nearest neighboring lattice sites (3D) or to one of the two neighboring lattice sites if it is sliding on the DNA (1D), which is represented by the green line of lattice sites. O obstacles (black) are placed on the DNA. Their dynamics depend on the type of obstacle (see the text), but in all cases they are not allowed to perform excursions to the bulk solution. Molecules have square geometry and diffusion is only allowed if the destination site is unoccupied. Note that the simulations use a three-dimensional lattice rather than the two-dimensional one depicted schematically here. (B) Search time (normalized by the search time $T_{\mathrm{s}}$ in the absence of non-specific binding of the DBP to the DNA) as a function of the sliding length $\lambda$. Data points are simulation results, and the line is the ratio between eqn (5) and $T_{\mathrm{s}}=V / 4 \mathrm{Da}$. (C) $T / T_{\mathrm{s}}$ for different DNA concentrations, plotted as a function of the unbinding rate $k_{\text {off. }}$ Different minimal search times and optimal unbinding rates are observed for different DNA concentrations. 
(since obstacles are not allowed to unbind, and moves within the bulk solution are always unhindered). Thus, no interactions other than steric repulsion are considered between the DBP and the obstacles.

If the DBP is bound to the DNA, it can either diffuse on the DNA or unbind from it with a rate $k_{\text {off }}$. If the DBP diffuses on the DNA, it performs a $1 \mathrm{D}$ random walk along the $y$ axis with diffusion constant $D_{1}$. Unless stated otherwise, we choose $D_{1}=D_{3} / 10$, as $1 \mathrm{D}$ diffusion is typically slower than $3 \mathrm{D}$ diffusion. ${ }^{9,11}$ Since we are interested in the effects that obstacles on the DNA have on the facilitated diffusion process, we implement three types of obstacles with different dynamics on the DNA: (i) static (immobile) obstacles, (ii) obstacles that diffuse on the DNA with a diffusion constant $D_{1}$, and (iii) obstacles that both diffuse and unbind from the DNA. Facilitated diffusion is expected to be affected differently by these three types of obstacles. 1D diffusion of both the DBP and the mobile obstacles is implemented as follows: if the destination site of a move is already occupied by another molecule, the move is rejected and the position is not updated. Position update of different molecules is performed in a random-sequential fashion: In every simulation step, we update the DBP position together with $O$ randomly chosen obstacles, in such a way that on average all particles are updated once per simulation step.

1D diffusion takes places until the DBP unbinds from the DNA with probability $P_{\text {off }}$. Thus, $P_{\text {off }}$ determines the rate at which the DBP unbinds from the DNA, $k_{\text {off }}=P_{\text {off }} \tau^{-1}$. We note that experimentally, the unbinding rate is typically varied via the salt concentration, ${ }^{23,24}$ because non-specific binding to DNA is typically of electrostatic nature and can be screened by large salt concentrations, resulting in large values of $P_{\text {off }}$.

In our simulations, the DBP finds the target either by 3D diffusion, if hopping from the bulk to one of the six neighboring lattice sites of destination happens to be the target, or by $1 \mathrm{D}$ diffusion if one of the two neighboring lattice sites is the target. Once the target has been found, we record the search time, and the DBP is randomly placed in the bulk solution.

\section{Facilitated diffusion without obstacles}

We start by considering the reference case of facilitated diffusion without obstacles on the DNA. This has been extensively studied in the past, both theoretically and computationally. ${ }^{13-18,25-27}$ In particular, it is well known that the efficiency of facilitated diffusion strongly depends on DNA concentration, $D_{1}$ and salt concentration $\left(k_{\text {off }}\right){ }^{26,50,54}$ The lattice model allows us to modulate these parameters and also provides a rather intuitive interpretation of the facilitated diffusion mechanism.

To begin with, we simulate facilitated diffusion in lattices of different sizes, thus effectively varying the DNA concentration. We keep the length of the simulation box (the direction parallel to the DNA) fixed at $m_{y}=1000 l$, but vary the box width in the other two dimensions $\left(m_{x}=m_{z}=L=10,20,30\right.$ and 60l). Simulations are run until 2000 target finding events have taken place.
In order to quantify the efficiency of facilitated diffusion in comparison to the free $3 \mathrm{D}$ diffusion case, we consider the ratio of the average search times $T / T_{\mathrm{s}}$. Here, $T$ is the average time for finding the target by facilitated diffusion, and $T_{\mathrm{s}}$ is the average time for finding the target via free 3D diffusion in the absence of DNA. Thus, if this ratio is less than one, facilitated diffusion is more efficient than the free diffusion case, whereas if the ratio exceeds one, it would be better for the system to find the target by simple 3D diffusion.

The essence of facilitated diffusion can be understood based on the following simple argument: the rate for target finding is diffusion-limited and thus given by $k=\gamma D a$, and the corresponding search time $T=(k c)^{-1}$, where $D$ is the diffusion coefficient, $a$ the size of the target, and $c=1 / V$ the concentration of the DBP (in our scenario a single one). $\gamma$ is a numerical prefactor, which is $\gamma=4 \pi$ in the classical Smoluchowski result, ${ }^{55}$ and $\gamma=4$ in our lattice model, where the target is a site on the DNA. Thus, the average time for target finding is obtained as $T=1 / k=V /(\gamma D a)$. The presence of the DNA has two different effects on the DBP dynamics, which can both be understood by introducing effective parameters: on one hand, transient binding to the DNA effectively slows down diffusion. Since diffusion along the DNA is typically slow, one can interpret binding to the DNA as pauses in 3D diffusion. These pauses can be incorporated into an effective diffusion coefficient

$$
D_{\text {eff }}=D_{3}\left(1-P_{\mathrm{b}}\right),
$$

where $P_{\mathrm{b}}$ is the probability for the DBP to be bound to the DNA. For the lattice model, this probability is given by

$$
P_{\mathrm{b}}=\frac{1}{1+\frac{\left(L^{2}-l^{2}\right) k_{\mathrm{off}}}{4 D_{3}}} .
$$

On the other hand, sliding along the DNA effectively increases the size of the target. To reach the target, the DBP does not need to bind to the target site directly, but may also bind close to the target and slide there by $1 \mathrm{D}$ diffusion. The average distance, over which the DBP slides along the DNA while bound, is given by

$$
\lambda=\left(\frac{2 D_{1}}{k_{\mathrm{off}}}\right)^{1 / 2}
$$

and is called the sliding length. Thus, the target size is effectively increased to $a_{\mathrm{eff}}=\lambda$.

Taken together, the two effects lead to

$$
\begin{aligned}
k & =\gamma D_{\mathrm{eff}} a_{\mathrm{eff}}=\gamma D_{3}\left(1-P_{\mathrm{b}}\right) \lambda \\
& =\gamma D_{3} \frac{1}{1+\frac{4 D_{3}}{\left[\left(L^{2}-l^{2}\right)\right] k_{\mathrm{off}}}} \times \sqrt{2 D_{1} / k_{\mathrm{off}}}
\end{aligned}
$$

and a search time of

$$
T=(k c)^{-1}=\frac{V}{\gamma D_{3} \lambda}+\frac{2 L_{\mathrm{c}} \lambda}{\gamma D_{1}} .
$$


In the last expression, we have used $c=1 / V$ and defined the contour length of the DNA $L_{\mathrm{c}}=V /\left(L^{2}-l^{2}\right)$ to bring it into the form derived by Halford and Marko, ${ }^{14}$ with which the last expression agrees up to the numerical prefactor $\gamma$. It is important to notice that only the latter of the two effects facilitates diffusion to the target, while the former slows it down, so the name 'facilitated diffusion' is only appropriate in a limited range of the parameter space. Since measured 1D diffusion coefficients are typically smaller than 3D diffusion coefficients, ${ }^{9,11}$ the slowing of diffusion has an important effect on non-specific binding to DNA and may well be the dominant effect for many proteins. Fig. 1B shows the $T / T_{\mathrm{s}}$ ratio as a function of $\lambda$ for constant $D_{1}$. As $\lambda$ increases, longer sliding events on the DNA take place when the DBP binds nonspecifically to the DNA. Thus, the effective target size increases and facilitated diffusion becomes optimal at $\lambda \simeq 11 \mathrm{l}$. For larger $\lambda$, the DBP spends more and more time non-specifically bound to the DNA, thereby $D_{\text {eff }}$ becomes smaller and facilitated diffusion becomes inefficient. A good agreement between our simulation data (red squares), and our analytical description (solid line) is observed. We note that the analytical argument given above is only valid for sliding lengths that exceed one lattice site. To correct this effect, we have used an effective target size $a_{\text {eff }}=\max \{a, \lambda\}$ for the solid line.

An important feature of this result is that the search time is non-monotonic as a function of the DNA binding strength. This feature has been a hallmark of facilitated diffusion, which has been at the center of many theoretical studies, ${ }^{6,7,14,15,17}$ and which was confirmed experimentally by varying the salt concentration. ${ }^{23,24}$ Based on our simple argument above, it can be interpreted as arising from the opposite effects of increasing the strength of non-specific binding on the effective diffusion coefficient and the effective target size.

Fig. 1C shows $T / T_{\mathrm{s}}$ as a function of $k_{\text {off }}$ for four different DNA concentrations. In all the cases, a non-monotonic behavior is observed as the value of $k_{\text {off }}$ is modulated. For low values of $k_{\text {off }}$, ratios larger than one are obtained, reflecting mostly the effective reduction in the $3 \mathrm{D}$ diffusion constant. Thus, in this parameter range, 3D diffusion in the absence of DNA is more effective that facilitated diffusion. For intermediate values of $k_{\text {off }}$, a minimum in the $T / T_{\mathrm{s}}$ ratio is observed. In this case, sliding on the DNA indeed facilitates binding to the promoter, due to the effectively larger target size, i.e. $\lambda+l$. For high values of $k_{\text {off }}$, the $T / T_{\text {s }}$ ratio gets close to one, as DNA binding is weak and has only a small effect.

In the case of $V=60 \times 1000 \times 60 l^{3}$, the optimal value of $k_{\text {off }}$ is found to be $0.0002 \tau^{-1}$. Then, the DBP diffuses on average over a sliding length of $\lambda \simeq 11 l$. With the obtained optimal value of $k_{\text {off }}$ we can obtain the probability that the DBP is bound to the DNA $P_{\mathrm{b}}=0.48$, see eqn (1). Thus, on average, the DBP spends approximately the same time bound to the DNA as diffusing freely in the bulk solution. This is in agreement with Marko's model, which predicts that the fraction of time the DBP spends on the DNA is the same fraction of time it spends in the bulk solution. ${ }^{14}$ Notably, the latter prediction is not general and in cases with high DNA concentration it is no longer valid. For example, when taking the case of $V=10 \times$ $1000 \times 10 l^{3}$, we find that the optimal unbinding rate is $k_{\text {off }}=$ $0.1 \tau^{-1}$. When substituting the latter value of $k_{\text {off }}$ into eqn (2), we obtain that $P_{\mathrm{b}}=0.063$. Here, the very frequent DBP-DNA nonspecific binding events drastically reduce $D_{3}$ if the DBP spends a relatively long time on the DNA. Thus, the optimal DBP dynamics is the one at which the DBP spends most of the time in the bulk solution with very short sliding events on the DNA, see the pink data in Fig. 1B.

Though similar qualitative behavior in facilitated diffusion is observed at all DNA concentrations, large quantitative differences are obtained. As the DNA concentration increases, the minimum in the $T / T_{\mathrm{S}}$ ratio gets close to one, and the optimal $k_{\text {off }}$ value shifts to the right. This shows that at large DNA concentrations, it is more efficient for the system to tune the $k_{\text {off }}$ parameter to large values, and thus, decrease $P_{\mathrm{b}}$.

Eqn (5) suggests that there are two relevant parameters of facilitated diffusion, the binding probability $P_{\mathrm{b}}$ and the sliding length $\lambda$. Each of these parameters determines the strength of one of the two effects of non-specific DNA binding. They are related to the microscopic parameters $k_{\text {off }}$ and $D_{1}$, but using these two parameters does not separate the two effects. Experimentally, modulation of the parameters via salt concentration modulates $k_{\text {off }}$ and thereby acts via both effects.

As a check of the parameter dependencies indicated by eqn (5), we systematically vary $P_{\mathrm{b}}$ and $\lambda$. This is done microscopically by modulating $k_{\text {off }}$ with the $D_{1} / k_{\text {off }}$ ratio fixed and $D_{1}$ for constant $k_{\text {off }}$, respectively. The results are shown in Fig. 2 . The color map in Fig. 2A shows the rate for target binding as a function of both parameters. Light blue color indicates parameter combinations for which 'facilitated diffusion' is indeed optimal, i.e. the combination of $3 \mathrm{D}$ and $1 \mathrm{D}$ diffusion is more efficient than free $3 \mathrm{D}$ diffusion $\left(T / T_{\mathrm{s}}<1\right)$. It is rather inefficient if the DBP remains bound to the DNA for long time without scanning the DNA. The dependencies on the individual parameters are shown in Fig. $2 \mathrm{~B}$ and $\mathrm{C}$, respectively. Fig. 2B shows the promoter finding rate as a function of the sliding length for constant values of $k_{\text {off }}$ and, thus, $P_{\mathrm{b}}$. The horizontal lines represent the limiting case where the DBP binds to the DNA but does not diffuse along it $\left(D_{1}=0\right)$. The star indicates the finding rate in the absence of DNA. As expected from eqn (5), the rate increases linearly with increasing sliding length (except for sliding lengths $\lambda \lesssim l$, where the effective target sizes becomes $\simeq l$ ). The linear increase is seen for all values of $k_{\text {off }}$. The plot also shows that sufficiently large sliding lengths are needed in order for the binding rate to exceed the free binding rate in the absence of DNA. The minimal required sliding length for facilitation increases with increasing $P_{\mathrm{b}}$ or decreasing $k_{\text {off }}$.

Fig. 2C shows the finding rate as a function of $\left(1-P_{\mathrm{b}}\right)$, the probability that the DBP is free in solution, for constant sliding length. For low values of $\left(1-P_{\mathrm{b}}\right)$, the DBP frequently binds non-specifically to the DNA. These events effectively reduce the $3 \mathrm{D}$ diffusion constant to $D_{\text {eff }}=D_{3}\left(1-P_{\mathrm{b}}\right)$. In agreement with this expectation, the finding rate increases linearly with the probability to be unbound, $1-P_{\mathrm{b}}$. 

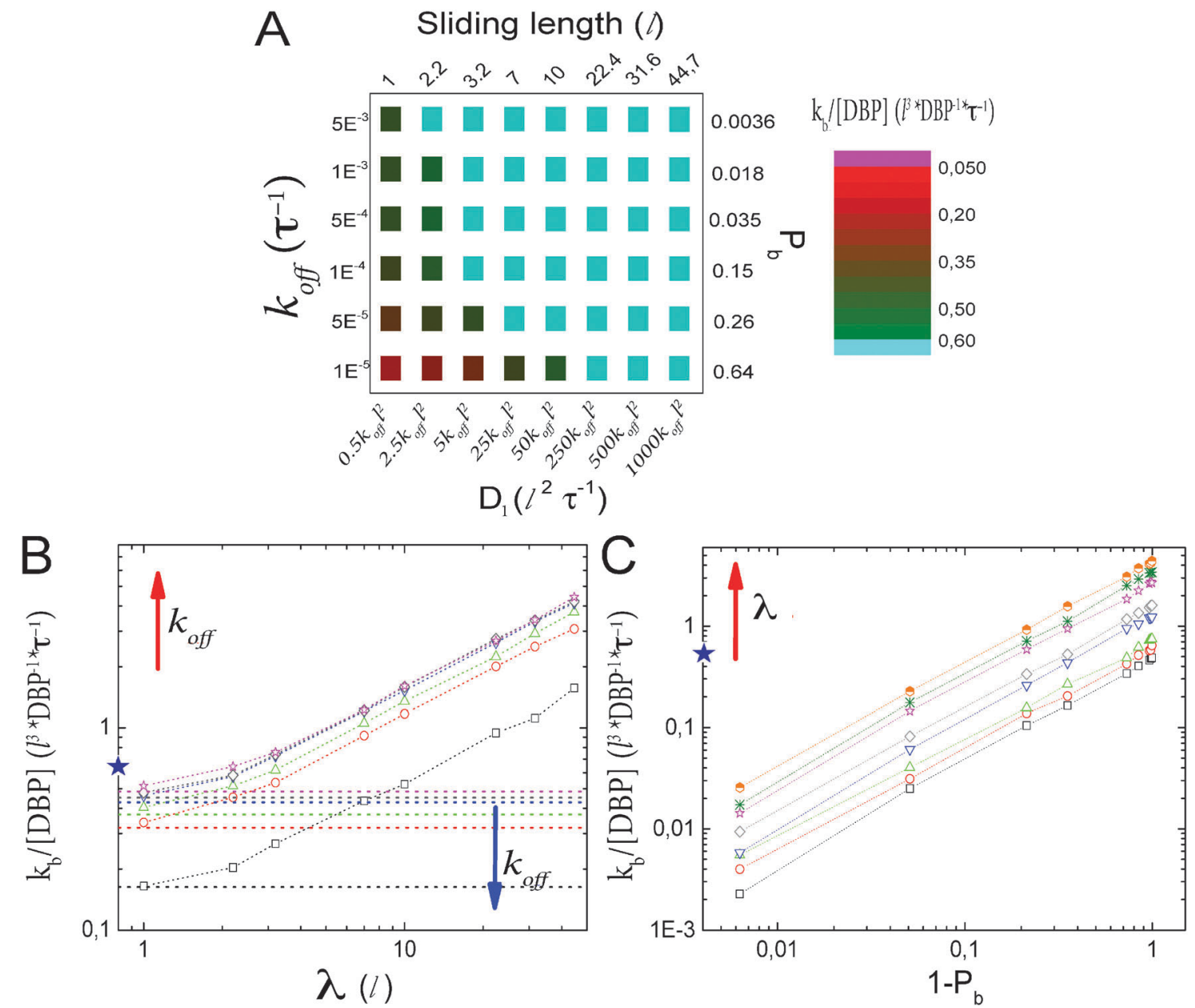

Fig. 2 Facilitated diffusion without obstacles on the DNA. (A) Target finding rate as a function of the two key parameters $P_{\mathrm{b}}$ and $\lambda$. Alternative axes show the microscopic parameters that are varied: $k_{\text {off }}$ and $D_{1} . k_{\text {off }}$ is varied with $D_{1} / k_{\text {off }}$ constant. Light blue data represent the set of parameters that leads to finding rates faster than the free 3D diffusion process. (B) Promoter finding rate as a function of $\lambda$ for different values of $k_{\text {off. }}$ For sufficiently large $\lambda$, the finding rate is linear in $\lambda$ and facilitated diffusion becomes more efficient than the free 3D diffusion process. Lines represent the limiting case where $D_{1}=0$, e.g. the DBP does not perform 1D diffusion on the DNA. (C) Finding rate as a function of the probability for the DBP to be unbound from the DNA $\left(1-P_{\mathrm{b}}\right)$ for different values of $\lambda$. For low values of $\left(1-P_{\mathrm{b}}\right)$, the finding rate increases linearly, in agreement with $k_{\mathrm{b}} /[\mathrm{L}] \propto D_{\text {eff }}=D_{3}\left(1-P_{\mathrm{b}}\right)$.

\section{Effect of obstacles on the DNA}

Next, we consider the impact that obstacles on the DNA have on facilitated diffusion. To that end, we implement three types of obstacles with different dynamics: obstacles of the first type diffuse on the DNA, but do not unbind. We set the crowders to diffuse on the DNA with the same 1D diffusion constant $D_{1}$ as the DBP. The obstacles can transiently occupy the target position, making it inaccessible for the DBP, independent of whether the DBP arrives via 3D diffusion or 1D sliding. The second type of obstacles that we consider are static $\left(D_{1}=0\right)$, with obstacles regularly spaced on the DNA. In this case, we make sure that the target is placed on a free DNA lattice site between the obstacles. Obstacles of type three also diffuse on the DNA, again with the same diffusion constant as the DBP, but, in addition, these obstacles can dissociate from the DNA with a rate $k_{\text {off }}^{\text {Obst }}$. In these simulations, we do not track diffusion of unbound obstacles in the bulk. Rather, when an obstacle dissociates from the DNA, we immediately place a new obstacle on a random position of the DNA in order to keep the DNA occupation fraction constant, see the representation of the obstacles in Fig. 3. The obstacle that dissociates is removed from the simulation.

Based on the two effects of (non-specific) binding to DNA discussed above, one can argue that obstacles on the DNA affect target finding only through the effective target size. By contrast, obstacles or crowders in the solution, which we do not consider here, would mostly affect it via the effective diffusion, although one can also expect an increase in the target size (due to crowding-enhanced binding to the DNA, which increases the sliding length $\left.{ }^{25,26}\right)$. Obstacles on the DNA that act as barriers for sliding effectively reduce the sliding length and thus the target size. Thus one can estimate an effective target size to be the smaller one of the two length scales of this system, the sliding length on one hand, and the average distance between obstacles on the other. 

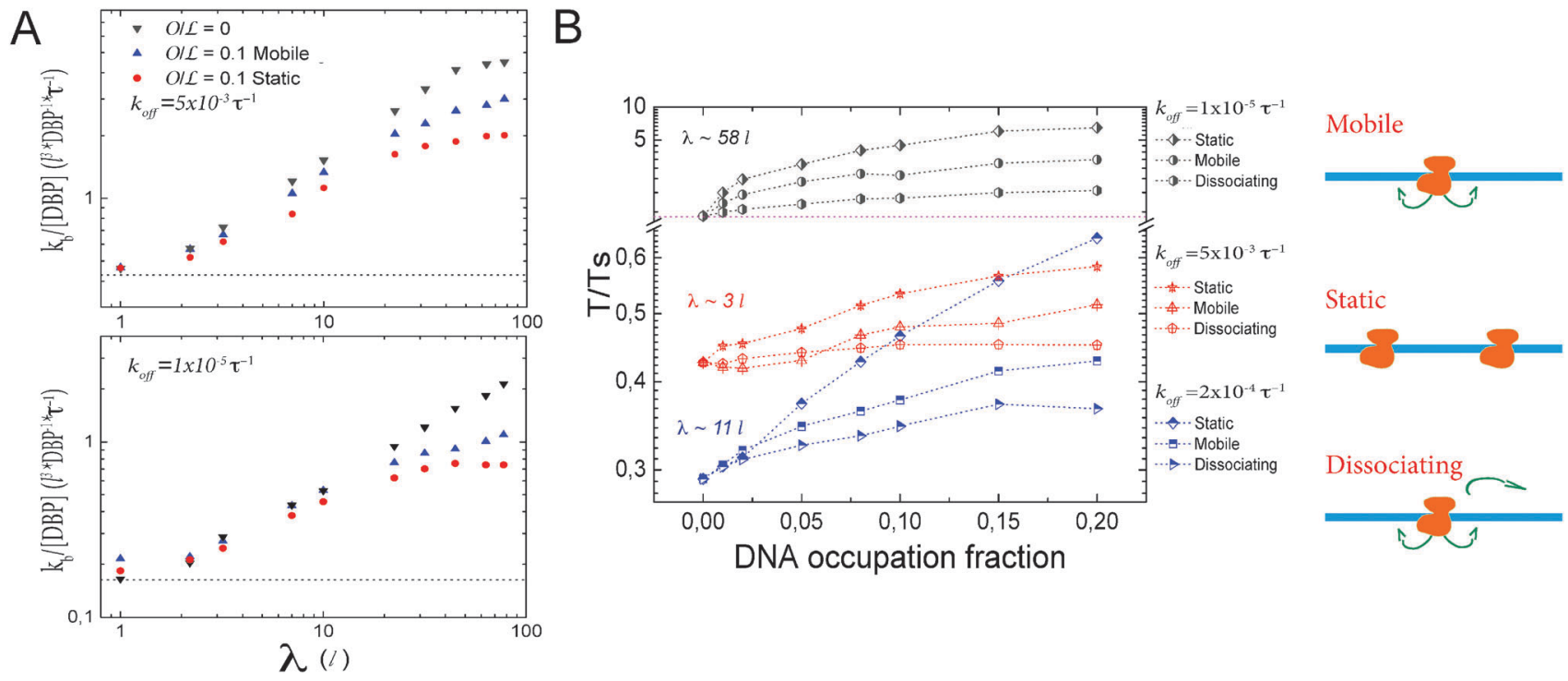

Fig. 3 Effects of obstacles on the DNA on facilitated diffusion. (A) Promoter finding rate as a function of $\lambda$ for different values of $k_{\text {off }}$ in the presence of obstacles on the DNA. In general, the presence of obstacles on the DNA hinders promoter finding. Depending on the type of obstacle, the binding rate is affected in a stronger or more moderate way. Lines represent the limiting case $D_{1}=0$, where the DBP does not diffuse on the DNA. (B) Dependence on obstacle coverage: search time ratio $T / T_{\mathrm{s}}$, as a function of the occupation fraction for three types of obstacles. Mobile obstacles perform 1D diffusion on the DNA, static obstacles are regularly distributed on the DNA and dissociating obstacles diffuse on the DNA and dissociate from it with an unbinding rate $k_{\text {off }}^{\text {obst }}=0.0002 \tau^{-1}$. Static obstacles have the strongest effects on facilitated diffusion, whereas obstacles that dissociate from the DNA have the smallest.

However, Fig. 3A shows that the dynamics of the roadblock also matter for the results. Here, we run simulations for two different values of $k_{\text {off }}, k_{\text {off }}=0.005 \tau^{-1}$ and $0.00001 \tau^{-1}$, at $10 \%$ DNA occupation. Shown data are for obstacle types one and two. The plot shows the target finding rate as a function of $\lambda$ when modulating $D_{1}$. Lines represent the limiting case of $D_{1}=0$. As $D_{1}$ increases, the finding rate becomes larger. For $\lambda \simeq 3 l$ the promoter finding rate starts to differ between the different types of obstacles. On one hand, at $O / \mathcal{L}=0.1$ there is an obstacle every 10 lattice sites if they are static. Thus, as $D_{1}$ increases, the search scenario becomes effectively one in which the DBP finds a target of size $a_{\mathrm{eff}}=10 l$, with an effective three dimensional diffusion constant $D_{\text {eff. }}$ On the other hand, if obstacles diffuse on the DNA, $a_{\text {eff }}$ fluctuates and can transiently be reduced or increased. Thus, saturation takes place at larger values of $\lambda$. We note that this average size depends on the obstacles' onedimensional diffusion constant.

In Fig. 3B we plot the $T / T_{\mathrm{s}}$ ratio as a function of the fractional occupation of the DNA by obstacles, for three different values of $k_{\text {off }}, k_{\text {off }}=0.005,0.0002$ and $0.00001 \tau^{-1}$. These values correspond to sliding lengths of $\lambda \simeq 3 l, 11 l$ and $58 l$, respectively. We note that in all cases, the presence of obstacles has a negative effect on facilitated diffusion, even for intermediate values of $k_{\text {off }}$ at low occupation fractions.

In general, static obstacles have the largest impact on facilitated diffusion, because they effectively trap the DBP once it has been bound non-specifically to the DNA. In this case the only way that the DBP can overcome an obstacle is to unbind from the DNA. Thus, the negative effect of static obstacles on the $T / T_{\mathrm{S}}$ ratio becomes larger as the unbinding rate $k_{\text {off }}$ decreases. Type- 1 obstacles have the second largest effect on facilitated diffusion. Here, even though obstacles trap the DBP in regions where the target may not be present, this trapping changes as the obstacles diffuse on the DNA, making the target accessible to the DBP, even if it remains bound to the DNA for long time. However, 1D diffusion is effectively slowed down by obstacles on the DNA. The effect of the obstacles decreases as the DBP unbinding rate increases. Type- 3 obstacles have the weakest effect on facilitated diffusion. In this case, the DBP can access the target in the same way as for type one. Since the obstacles can unbind from the DNA, the trapping effect is weaker and the DBP can scan larger sections of DNA. Thus, the impact of this type of obstacle on facilitated diffusion is more modest.

Interestingly, for different types of obstacles and DBP unbinding rates, the $T / T_{\mathrm{S}}$ ratios can get close and even cross (Fig. 3B). To take a better look into this issue, we reconstruct the facilitated diffusion plot for different levels of fractional DNA occupation, and obtain the $k_{\text {off }}$ value at which facilitated diffusion is optimal, see Fig. 4A-D. As can be observed, the minimum shifts towards higher values of $k_{\text {off }}$ and higher values of $T / T_{\mathrm{S}}$. Next, we plot in Fig. $4 \mathrm{E}$ the optimal values of the $T / T_{\mathrm{s}}$ ratio as a function of DNA occupation. Static obstacles have the strongest effects on facilitated diffusion, and the dissociating obstacles the weakest. In Fig. $4 \mathrm{~F}$, we plot the corresponding values of $k_{\text {off }}$ at which facilitated diffusion is the most effective. Here, as the DNA occupation increases, the DBP needs to bind less tightly and scan smaller sections of DNA at every 1D sliding excursion. This suggests that in order to be as efficient as possible, the DBP has to spend more time in the bulk solution 

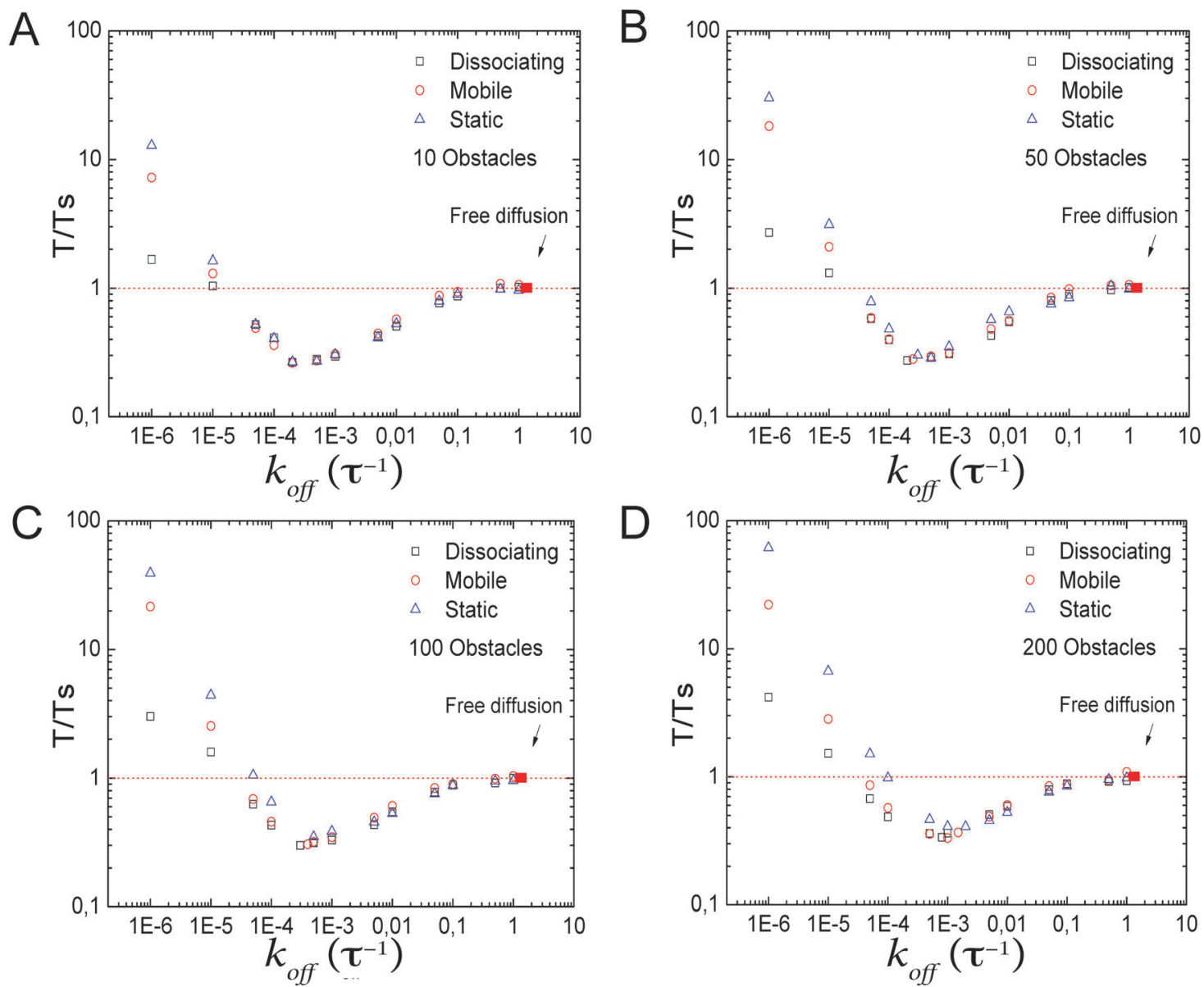

D
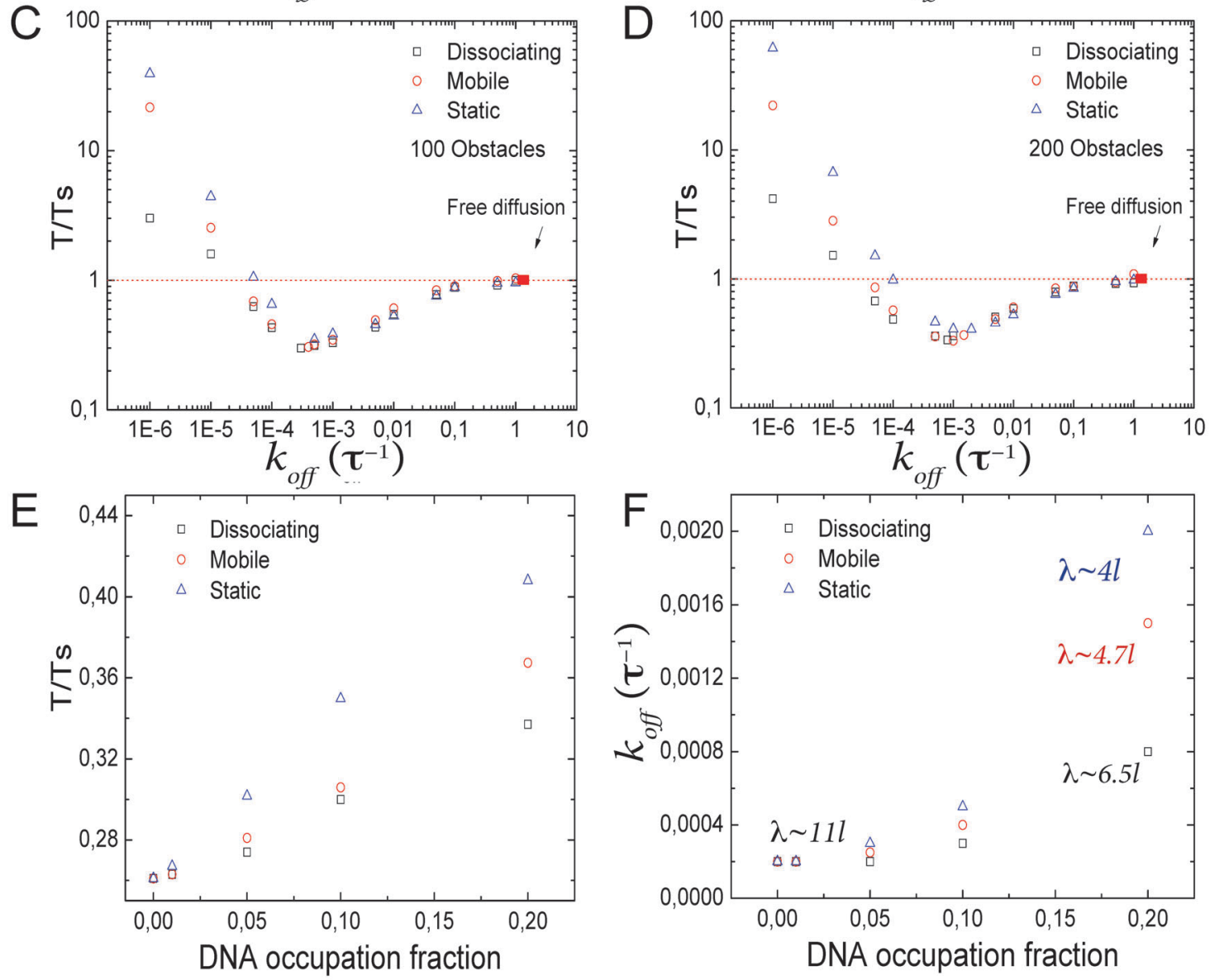

Fig. 4 Facilitated diffusion in the presence of obstacles on the DNA. (A-D) Search time ratio $T / T_{\mathrm{s}}$ as a function of $k_{\text {off }}$ for the three types of obstacles. As the DNA occupation fraction increases, the minimum in $T / T_{\mathrm{s}}$ changes, as well as the value of $k_{\text {off }}$ where it occurs. (E) Minimal search time as a function of the DNA occupation fraction. As the number of obstacles increases, facilitated diffusion becomes less efficient, with the static obstacles affecting facilitated diffusion the most. (F) Corresponding optimal value of $k_{\text {off }}$ as a function of the DNA occupation fraction. In order to be as efficient as possible, the DBP must spend more time in the bulk solution, to avoid being trapped between obstacles.

than on the DNA. Specifically, we showed above that in the absence of obstacles on the DNA, the DBP spends on average $\simeq 50 \%$ of the total search time bound to the DNA. In the presence of static obstacles occupying $20 \%$ of the DNA, the average time the DBP spends bound to the DNA under optimal conditions is decreased to only $9 \%$.

\section{Concluding remarks}

In this paper, we used a simple computational approach to study the promoter finding process via facilitated diffusion. We used lattice simulations to revisit how the promoter finding process is affected when modulating different parameters in 
our simulations. Non-specific binding to the DNA effectively reduces diffusion in the bulk and thereby the diffusion-limited binding to the target. At the same time, sliding along the DNA effectively increases the size of the target. The competition of these two effects leads to a characteristic maximum in the binding rate. This maximum is very sensitive to the concentration of DNA, $D_{1}, k_{\text {off }}$ and $\lambda$. The lattice model provides a rather intuitive picture of these situations as well as an efficient computational implementation of facilitated diffusion.

In addition, we have studied the effects that different types of obstacles have on the facilitated diffusion process. In general, since the DBP is (at least transiently) trapped between two obstacles, its sliding length is reduced, decreasing effectively the target size. Beyond that, our results suggest that obstacles with different dynamics on the DNA have different effects on facilitated diffusion, with static obstacles affecting the finding rate the most. In order to be as efficient as possible, the DBP has to modulate its interaction with the DNA, and spend more time in the bulk solution. The methods we used here can be applied more generally and there are a number of possible extensions to this work, such as addressing the effects of crowders on the bulk solution on the facilitated diffusion process. Other aspects of facilitated diffusion will require more detailed molecular approaches, for example the effect of DNA flexibility and supercoiling, which are difficult to implement in the lattice model. The latter has been shown to have an effect on target search kinetics with more rapid target finding for a coiled DNA conformation than for stretched DNA conformations. ${ }^{56-58}$ Studying the interplay of the DNA conformation and obstacles will be a task for future work.

\section{References}

1 D. F. Browning and S. J. Busby, The regulation of bacterial transcription initiation, Nat. Rev. Microbiol., 2004, 2(1), 57-65.

2 G. D. Stormo, DNA binding sites: representation and discovery, Bioinformatics, 2000, 16(1), 16-23.

3 U. Gerland, J. D. Moroz and T. Hwa, Physical constraints and functional characteristics of Transcription Factor-DNA interaction, Proc. Natl. Acad. Sci. U. S. A., 2002, 99(19), 12015-12020.

4 F. Wang, S. Redding, I. J. Finkelstein, J. Gorman, D. R. Reichman and E. C. Greene, The promoter-search mechanism of Escherichia coli RNA polymerase is dominated by three-dimensional diffusion, Nat. Struct. Mol. Biol., 2013, 20(2), 174-181.

5 A. Riggs, S. Bourgeois and M. Cohn, The lac repressor-operator interactions. III. Kinetic studies, J. Mol. Biol., 1970, 53, 401.

6 O. G. Berg, R. B. Winter and P. H. von Hippel, Diffusiondriven mechanisms of protein translocation on nucleic acids. 1. Models and theory, Biochemistry, 1981, 20, 6929-6948.

7 P. H. von Hippel and O. G. Berg, Facilitated target location in biological systems, J. Biol. Chem., 1989, 264, 675-678.

8 S. G. Erskine and S. E. Halford, Reactions of the EcoRV restriction endonuclease with fluorescent oligodeoxynucleotides: identical equilibrium constants for binding to specific and non-specific DNA, J. Mol. Biol., 1998, 275, 759-772.
9 Y. M. Wang, R. H. Austin and E. C. Cox, Single Molecule Measurements of Repressor Protein 1D Diffusion on DNA, Phys. Rev. Lett., 2006, 97, 048302.

10 S. Cranz, C. Berger, A. Baici, I. Jelesarov and H. R. Bosshard, Monomeric and dimeric bZIP transcription factor GCN4 bind at the same rate to their target DNA site, Biochemistry, 2004, 43, 718-727.

11 J. Elf, G.-W. Li and X. S. Xie, Probing transcription factor dynamics at the single-molecule level in a living Cell, Science, 2007, 316(5828), 1191-1194.

12 P. Hammar, P. Leroy, A. Mahmutovic, E. G. Marklund, O. G. Berg and J. Elf, The lac repressor displays facilitated diffusion in living cells, Science, 2012, 336(6088), 1595-1598.

13 I. M. Sokolov, R. Metzler, K. Pant and M. C. Williams, Target search of N sliding proteins on a DNA, Biophys. J., 2005, 89, 895.

14 S. E. Halford and J. F. Marko, How do site-specific DNAbinding proteins find their targets?, Nucleic Acids Res., 2004, 32(10), 3040-3052.

15 M. Bauer and R. Metzler, Generalized facilitated diffusion model for DNA-binding proteins with search and recognition states, Biophys. J., 2012, 102, 2321.

16 K. V. Klenin, H. Merlitz, J. Langowski and C.-X. Wu, Facilitated diffusion of DNA-binding proteins, Phys. Rev. Lett., 2006, 96, 018104.

17 M. Slutsky and L. A. Mirny, Kinetics of protein-DNA interaction: facilitated target location in sequence-dependent potential, Biophys. J., 2004, 87(6), 4021-4035.

18 A. B. Kolomeisky, Physics of protein-DNA interactions: mechanisms of facilitated target search, Phys. Chem. Chem. Phys., 2013, 13, 2088-2095.

19 H. Kabata, O. Kurosawa, I. Arai, M. Washizu, S. A. Margarson, R. E. Glass and N. Shimamoto, Visualization of single molecules of RNA polymerase sliding along DNA, Science, 1993, 262(5139), 1561-1563.

20 N. P. Stanford, M. D. Szczelkun, J. F. Marko and S. E. Halford, One- and three-dimensional pathways for proteins to reach specific DNA sites, EMBO J., 2000, 19(23), 6546-6557.

21 S. Reddinga and E. C. Greene, How do proteins locate specific targets in DNA?, Chem. Phys. Lett., 2013, 570, 1-11.

22 L. J. Friedman, J. P. Numm and J. Gelles, RNA polymerase approaches its promoter without long-range sliding along DNA, Proc. Natl. Acad. Sci. U. S. A., 2013, 110(24), 9740-9745.

23 R. B. Winter, O. G. Berg and P. H. von Hippel, Diffusion-driven mechanisms of protein translocation on nucleic acids. 3 . The Escherichia coli lac repressor-operator interaction: kinetic measurements and conclusions, Biochemistry, 1981, 20, 6961-6977.

24 D. M. Gowers, G. G. Wilson and S. E. Halford, Measurementof the contributions of $1 \mathrm{D}$ and $3 \mathrm{D}$ pathways to the translocation of a protein along DNA, Proc. Natl. Acad. Sci. U. S. A., 2005, 102, 15883-15888.

25 C. A. Brackley, M. E. Cates and D. Marenduzzo, Intracellular Facilitated Diffusion: Searchers, Crowders, and Blockers, Phys. Rev. Lett., 2013, 111, 108101.

26 R. K. Das and A. B. Kolomeisky, Facilitated search of proteins on DNA: correlations are important, Phys. Chem. Chem. Phys., 2010, 12, 2999-3004. 
27 A. Marcovitz and Y. Levy, Sliding dynamics along DNA: a molecular perspective, Innovations in Biomolecular Modeling and Simulation, RSC, Biomolecular Sciences 24, 2012, ch. 10, pp. 237-262.

28 S. B. Zimmerman and S. O. Trach, Estimation of macromolecule concentrations and excluded volume effects for the cytoplasm of Escherichia coli, J. Mol. Biol., 1991, 222, 599-620.

29 D. S. Cayley and M. T. Record Jr, Large changes in cytoplasmic biopolymer concentration with osmolality indicate that macromolecular crowding may regulate proteinDNA interactions and growth rate in osmotically stressed Escherichia coli K-12, J. Mol. Recognit., 2004, 17, 488-496.

30 N. Muramatsu and A. P. Minton, Tracer diffusion of globular proteins in concentrated protein solutions, Proc. Natl. Acad. Sci. U. S. A., 1988, 85, 2984-2988.

31 J. Han and J. Herzfeld, Macromolecular diffusion in crowded solutions, Biophys. J., 1993, 65, 1155-1161.

32 J. S. Kim and A. Yethiraj, Effects of macromolecular crowding on reaction rates: a computational and theoretical study, Biophys. J., 2009, 96, 1333-1340.

33 D. Gomez and S. Klumpp, Biochemical reactions in crowded environments: revisiting the effects of volume exclusion with simulations, Front. Phys., 2015, 3, 45.

34 B. K. Derham and J. J. Harding, The effect of the presence of globular proteins and elongated polymers on enzyme activity, Biochim. Biophys. Acta, 2006, 1764, 1000-1006.

35 M. G. S. Norris and N. Malys, What is the true enzyme kinetics in the biological system? An investigation of macromolecular crowding effect upon enzyme kinetics of glucose6-phosphatase dehydrogenase, Biochem. Biophys. Res. Commun., 2011, 405, 388-392.

36 H.-X. Zhou, G. Rivas and A. P. Minton, Macromolecular crowding and confinement: Biochemical, biophysical, and potential physiological consequences, Annu. Rev. Biophys., 2008, 37, 375-397.

37 B. van den Berg, R. J. Ellis and C. M. Dobson, Effects of macromolecular crowding on protein folding and aggregation, EMBO J., 1999, 18(24), 6927-6933.

38 M. S. Cheung, D. Klimov and D. Thirumalai, Molecular crowding enhances native state stability and refolding rates of globular proteins, Proc. Natl. Acad. Sci. U. S. A., 2005, 102(13), 4753-4758.

39 J. Mittal and R. B. Best, Dependence of Protein Folding Stability and Dynamics on the Density and Composition of Macromolecular Crowders, Biophys. J., 2010, 98, 315-320.

40 D. Gnutt, M. Gao, O. Bryski, M. Heyden and S. Ebbinghaus, Effekte des Volumenausschlusses in lebenden Zellen, Angew. Chem., 2015, 127, 2578-2581.

41 H. Matsuda, G. G. Putzel, V. Backman and I. Szleifer, Macromolecular crowding as a regulator of gene transcription, Biophys. J., 2014, 106, 1801-1810.
42 M. J. Morelli, R. J. Allen and P. R. ten Wolde, Effects of macromolecular crowding on genetic networks, Biophys. J., 2011, 101, 2882-2891.

43 C. Tan, S. Saurabh, M. P. Bruchez, R. Schwartz and P. LeDuc, Molecular crowding shapes gene expression in synthetic cellular nanosystems, Nat. Nanotechnol., 2013, 8, 602-608.

44 S. P. Haugen, W. Ross and R. L. Gourse, Advances in bacterial promoter recognition and its control by factors that do not bind DNA, Nat. Rev. Microbiol., 2008, 6, 507-519.

45 E. Nudler, RNA polymerase active center: the molecular engine of transcription, Annu. Rev. Biochem., 2009, 78, 335-361.

46 R. M. Saecker, M. T. Record and P. L. Dehaseth, Mechanism of bacterial transcription initiation: RNA polymerasepromoter binding, isomerization to initiation-competent open complexes, and initiation of RNA synthesis, J. Mol. Biol., 2011, 412, 754-771.

47 C. L. Peterson and M. A. Laniel, Histones and histone modifications., Curr. Biol., 2004, 14(14), R546-R551.

48 S. C. Dillon and C. J. Dorman, Bacterial nucleoid-associated proteins, nucleoid structure and gene expression, Nat. Rev. Microbiol., 2010, 8, 185-195.

49 Y. Meroz, I. Eliazar and J. Klfter, Facilitated diffusion in a crowded environment: from kinetics to stochastics, J. Phys. A: Math. Theor., 2009, 42, 434012.

50 A. Marcovitz and Y. Levy, Obstacles may facilitate and direct DNA search by proteins, Biophys. J., 2013, 104, 2042-2050.

51 G.-W. Li, O. G. Berg and J. Elf, Effects of macromolecular crowding and DNA looping on gene regulations kinetics, Nat. Phys., 2009, 5, 294-297.

52 A. Mahmutovic, O. G Berg and J. Elf, What matters for lac repressor search in vivo - Sliding, Hopping, Intersegment Transfer, Crowding on DNA or Recognition?, Nucleic Acids Res., 2015, 43(7), 3454-3464.

53 L. Liu and K. Luo, Molecular crowding effect on dynamics of DNA-binding proteins search for their targets, J. Chem. Phys., 2014, 141, 225102.

54 O. Givaty and Y. Levy, Protein Sliding Along DNA: Dynamics and Structural Characterization, J. Mol. Biol., 2009, 385, 1087-1097.

55 M. V. Smoluchowski, Versuch einer mathematischen Theorie der Koagulationskinetik kolloider Lösungen, Z. Phys. Chem., 1917, 92, 129-168.

56 B. van den Broek, M. A. Lomholt, S.-M. J. Kalisch, R. Metzler and G. J. L. Wuite, How DNA coiling enhances target localization by proteins, Proc. Natl. Acad. Sci. U. S. A., 2008, 105(41), 15738-15742.

57 D. M. Gowers and S. E. Halford, Protein motion from nonspecific to specific DNA by three-dimensional routes aided by supercoiling, EMBO J., 2003, 22, 1410-1418.

58 M. A. Lomholt, B. van den Broek, S. M. Kalisch, G. J. Wuite and R. Metzler, Facilitated diffusion with DNA coiling, Proc. Natl. Acad. Sci. U. S. A., 2009, 106(20), 8204-8208. 